\title{
The impact of intelligent automation in internal supply chains
}

\section{Tiago Coito*, Joaquim L. Viegas, Miguel S.E. Martins and Bernardo Firme}

IDMEC,

Instituto Superior Técnico,

Universidade de Lisboa,

Lisboa, Portugal

Email: tiagoascoito@tecnico.ulisboa.pt

Email: joaquim.viegas@tecnico.ulisboa.pt

Email: miguelsemartins@tecnico.ulisboa.pt

Email: bernardo.firme@tecnico.ulisboa.pt

*Corresponding author

\section{João Figueiredo}

IDMEC,

Universidade de Évora,

Évora, Portugal

Email: jfig@uevora.pt

\section{Susana M. Vieira}

IDMEC,

Instituto Superior Técnico,

Universidade de Lisboa,

Lisboa, Portugal

Email: susana.vieira@tecnico.ulisboa.pt

\section{João Miguel da Costa Sousa}

IDMEC,

Instituto Superior Técnico,

Universidade de Lisboa,

Lisboa, Portugal

Email: jmsousa@tecnico.ulisboa.pt

\begin{abstract}
Nowadays, industry is being forced to produce smaller and more diverse batches, increasing the complexity of internal supply chains. Data has become a valuable asset, supporting the development of intelligent automation solutions. Decision support systems, which leverage data, require the automation pyramid to be more flexible, as information needs to be exchanged simultaneously and in real-time with all automation layers. This paper proposes a framework for intelligent automation to deal with current challenges in
\end{abstract}

